\title{
Prospective comparison of the Gen-probe PACE 2 Assay and the Abbott Ligase Chain Reaction for the direct detection of Chlamydia trachomatis in a low prevalence population
}

\author{
KIRK M. DOING*†, KRISTOPHER CURTIS*, JENNIFER W. LONG† and MARGARET L. VOLOCK $\dagger$ \\ * Department of Microbiology, Biochemistry and Molecular Biology, University of Maine, Orono, ME 04469 \\ and †Affiliated Laboratory Inc., 925 Union Street, Suite 4, Bangor, ME 04401, USA
}

\begin{abstract}
In a prospective study, the Gen-Probe PACE 2 (GP) assay was compared with Abbott Laboratories' ligase chain reaction (LCR) assay for the detection of Chlamydia trachomatis. A total of 493 female patients consented to collection of two cervical samples; a first-void urine (FVU) sample was collected also from 446 of the participants. Cervical samples were tested by both GP and LCR; 16 samples (3.1\%) tested positive by both methods and no discrepant results were observed. All but one of the FVU samples collected from patients with a positive cervical sample was positive for $C$. trachomatis by LCR. The stability of FVU samples over time in the LCR test was also evaluated and proved to be significantly longer than the 4 days stated by the manufacturer. While LCR proved to be highly sensitive in detecting chlamydial infection in FVU samples, no difference was noted between LCR and GP in the detection of cervical $C$. trachomatis infection in this study population.
\end{abstract}

\section{Introduction}

Chlamydia trachomatis infections are among the most common sexually transmitted diseases in the USA, with an estimated four million new cases occurring annually at a cost approaching $\$ 2.4$ billion [1]. Urethritis is the most frequent infection in males. Cervicitis is the most common presentation in females, with approximately one-third of infected females having concomitant urethritis. Untreated, chlamydial infections can ascend the genitourinary tract, resulting in sequelae such as epididymitis in males and endometritis, salpingitis and pelvic inflammatory disease, which can lead to infertility, in women [2]. The high percentage of asymptomatic infections (75\% in females and $50 \%$ in males) is a significant public health concern and results in a large reservoir of infected individuals who continue to spread the disease. The potential for serious sequelae, the high numbers of infected individuals, and the high rate of asymptomatic infections underscore the need for an

Received 5 May 1998; revised version accepted 26 Aug. 1998.

Corresponding author: Dr K. M. Doing. efficient, cost-effective method for diagnosing chlamydial infection. Extensive screening of high-risk individuals for infection and intervention with appropriate therapy has been proposed, and in some cases demonstrated to be a cost-effective strategy in limiting transmission and preventing the serious sequelae, thus lowering health care costs overall $[3,4]$.

A DNA probe hybridisation assay is currently one of the most commonly used methods for the direct detection of $C$. trachomatis from urethral and cervical samples. Recently, a new generation of diagnostic tests that employ nucleic acid amplification for the detection of $C$. trachomatis has become available for routine use in the clinical laboratory. The increased sensitivity of these newer tests enables first void urine (FVU) to be tested in addition to the more traditional endocervical and urethral samples. In a prospective study, the Ligase Chain Reaction (LCR) amplification assay (Abbott Laboratories, Chicago, IL, USA) was compared with the Gen-Probe PACE 2 hybridisation assay (Gen-Probe, San Diego, CA, USA) for the direct detection of $C$. trachomatis from female cervical samples. In addition, FVU samples were tested by LCR, and the stability of positive urine samples was examined. 


\section{Materials and methods}

\section{Study population}

Patients aged 13-59 years (mean 22.6 years) were selected from four clinics: a university health centre, a local STD clinic, a community health care centre and a women's health clinic. Each participant was sexually active with a history of two or more sexual partners in the previous 2 years. Cervical samples from each patient were tested for $C$. trachomatis with both the Gen-Probe PACE 2 hybridisation assay (GP) and the Abbott LCR amplification assay.

\section{Sample collection and processing}

Two endocervical samples were collected from each patient with collection kits provided by the assay manufacturers. Practitioners were instructed to collect the samples in random order, following the instructions included with each sample collection kit. Samples were held at $2-20^{\circ} \mathrm{C}$ and transported to the laboratory on the day of collection. Upon receipt at the laboratory, LCR specimens were frozen at $-70^{\circ} \mathrm{C}$ until testing, which was done within 30 days of receipt.

Each GP sample was briefly vortex mixed and centrifuged for $5 \mathrm{~min}$ at $1200 \mathrm{~g}$. After centrifugation, $40 \mu \mathrm{l}$ of a mucolytic agent, provided by Gen-Probe, was added. The sample tube was then incubated at $60^{\circ} \mathrm{C}$ in a waterbath for $10 \mathrm{~min}$, followed by removal of $100 \mu \mathrm{l}$ with a pipette fitted with an extended-length barrier tip. This sample was considered free of extraneous DNA contamination and was stored frozen at $-70^{\circ} \mathrm{C}$ for use in the event that discrepant analysis was needed.

FVU for LCR amplification was requested from participants who had not voided in the previous hour $(\mathrm{n}=446)$. The first $15-25 \mathrm{ml}$ of urine were collected in a sterile plastic container. Urine samples were refrigerated immediately at $2-8^{\circ} \mathrm{C}$ and were transported to the laboratory on the day of collection. Within $24-48 \mathrm{~h}$ of receipt at the laboratory, $1.0 \mathrm{ml}$ was taken from each urine specimen, placed in a 1.5 $\mathrm{ml}$ microcentrifuge tube and frozen at $-70^{\circ} \mathrm{C}$ until tested. The remainder of each urine sample was stored at $2-8^{\circ} \mathrm{C}$; some of these samples were used in specimen stability studies, as described below.

\section{Gen-Probe}

The GP PACE 2 assay was completed as described previously [5]. Briefly, this involved pipetting $100 \mu \mathrm{l}$ of the sample into a $12 \times 75-\mathrm{mm}$ polystyrene reaction tube followed by the addition of $100 \mu$ l of DNA probe with a chemiluminescent label, complementary to rRNA sequences of the target organism. Hybridisation was allowed to proceed at $60^{\circ} \mathrm{C} \pm 1^{\circ} \mathrm{C}$ for $1 \mathrm{~h}$, followed by the addition of $1.0 \mathrm{ml}$ of separation reagent containing magnetic particles and a hydrolysis reagent.
DNA:RNA hybrids were separated with a magnetic tray and detected with a luminometer which expressed results in relative light units (RLU). Following previously established laboratory protocol, samples with results exceeding the positive cut-off point, but with RLUs below 1500 , were confirmed as 'true' positive samples with a probe competition assay (PCA) furnished by Gen-Probe.

\section{$L C R$}

The LCR assay targets DNA sequences located on a cryptic plasmid present in most strains of $C$. trachomatis, often in multiple copies. Samples for LCR testing were thawed and tested in batches following the guidelines outlined by the manufacturer. Frozen urine samples were thawed and then centrifuged at $9000 \mathrm{~g}$ for $15 \mathrm{~min}$. The supernate was removed immediately and the pellet was resuspended in $1.0 \mathrm{ml}$ of resuspension buffer containing $\geqslant 50 \mathrm{mM} \mathrm{MgCl}_{2}$, provided in a urine processing kit (Abbott Laboratories). All samples, cervical and processed urines, were heated at $97^{\circ} \mathrm{C} \pm 2^{\circ} \mathrm{C}$ for $15 \mathrm{~min}$ in a heat block, followed by cooling at room temperature for $15 \pm 5 \mathrm{~min}$. Swabs were removed from cervical samples and new caps were attached. One hundred $\mu 1$ of each heated sample were pipetted into an amplification vial supplied with the Abbott LCR test kits. Each amplification vial contained $100 \mu \mathrm{l}$ of the LCR reaction mix, consisting of the four oligonucleotide probes, thermostable Thermus thermophilus polymerase, Thermus DNA ligase, NAD, $\mathrm{Mg}^{2+}$, dCTP and dTTP in a $\mathrm{pH} 7.8$ buffer. The amplification vials were immediately placed into a thermocycler (Abbott Laboratories) and amplified as follows: 40 cycles at $93^{\circ} \mathrm{C}$ for $1 \mathrm{~s}, 59^{\circ} \mathrm{C}$ for $1 \mathrm{~s}$ and $62^{\circ} \mathrm{C}$ for $70 \mathrm{~s}$. Detection of amplified product was completed in an automated analyser $\left(\mathrm{LC}_{\mathrm{x}}\right)$ based on a microparticle EIA format that uses capture and detection antibodies specific for different haptens present on the oligonucleotide probes. A test result, positive or negative, expressed as a sample/cut-off (S/CO) ratio, is calculated by the instrument and ratios $>1.0$ are considered positive according to the manufacturer's instructions. Specimens with $\mathrm{S} / \mathrm{CO}$ values between 0.8 and 1.0 are deemed uninterpretable and the test is repeated. Repeat $\mathrm{S} / \mathrm{CO}$ values $\geqslant 1.0$ are then considered positive, and negative if $<1.0$. Controls and calibrators were included with each carousel, as directed by the manufacturer.

\section{Urine stability studies}

The package insert for the LCR assay states that FVU samples must be refrigerated immediately after collection, sent to the testing laboratory within $24 \mathrm{~h}$ of collection and then tested within 4 days. To determine if positive samples remain stable for longer periods of time, nine positive urine samples were refrigerated and re-tested by LCR at regular intervals over a period of 6 weeks. A $1.0-\mathrm{ml}$ volume from each stored urine sample 
was removed and processed on the day of testing as previously described.

The number of $C$. trachomatis elementary bodies (EB) present in each of the nine positive urine samples was determined by immunofluorescence. A $1.0-\mathrm{ml}$ volume from each positive urine sample was centrifuged at $9000 \mathrm{~g}$ for $15 \mathrm{~min}$, the supernate was discarded and the pellet was resuspended in $100 \mu \mathrm{l}$ of urine resuspension buffer (Abbott Laboratories). This suspension was cytocentrifuged at $2000 \mathrm{rpm}$ for $5 \mathrm{~min}$ (Shandon Cytospin 3, Shandon-Lipshaw, Pittsburgh, PA, USA). Slides were allowed to dry in air, fixed in methanol and stained for $15 \mathrm{~min}$ with $15 \mu \mathrm{l}$ of fluoresein-labelled monoclonal antibody specific for C. trachomatis major outer-membrane protein (Syva, San Jose, CA, USA). Smears were examined at magnifications of 500 and 1000 with a fluorescent microscope equipped with appropriate filters, and EB were counted.

\section{Results}

Of the 493 cervical samples tested, $16(3.2 \%)$ were positive for $C$. trachomatis. Interestingly, there were no discrepant results between GP and LCR. Four of the positive GP samples had values that exceeded the positive threshold but were under 1500 RLUs. Three of those samples were stored in sufficient quantities to be tested with the PCA assay, which confirmed all three to be 'true' positives.

Overall, 14 FVU samples $(3.2 \%)$ of the 446 tested positive by LCR for $C$. trachomatis. In all cases, the positive urine samples were from patients who also had positive cervical samples. One urine sample, collected from a patient whose cervical sample was positive for $C$. trachomatis by both GP and LCR, was negative by LCR; however, the initial $\mathrm{S} / \mathrm{CO}$ value of 0.55 was much higher than that noted for other negative samples (negative mean $\mathrm{S} / \mathrm{CO}=0.04$ ). LCR was repeated on this sample, and a negative but still elevated $\mathrm{S} / \mathrm{CO}$ value of 0.45 was obtained.
Five additional samples (two urine and three cervical) had initial S/CO values between 0.34 and 0.74 . Testing was repeated to determine if low-level amplification or inhibition of amplification was occurring in these samples. The resulting $\mathrm{S} / \mathrm{CO}$ values of $\leqslant 0.05$ indicated that all five samples were negative.

Of the nine urine samples positive for $C$. trachomatis by LCR that were re-tested at regular intervals, all except three remained positive over 6 weeks of testing (Table 1). One sample continued to test positive after 10 weeks (data not shown). Of the three samples reverting to negative, one (no. 491) tested negative after 3 weeks, but was positive at 4,5 and 6 weeks. The remaining two samples (nos. 361 and 511) tested positive until week 5 . The numbers of EB present in all nine samples, as determined by DFA staining of cytocentrifuge preparations, varied from zero to eight (Table 1). There was no evident correlation between the number of EB present and the length of time a specimen tested positive or reverted to negative.

\section{Discussion}

The comparison of commonly used assays for direct detection of $C$. trachomatis with recently introduced amplification-based methods is important as laboratories attempt to make clinical and budgetary decisions. LCR testing of cervical samples proved no more sensitive than the GP assay for the detection of $C$. trachomatis in this study population. To some degree, this was an unexpected finding, given the reported differences in sensitivity between direct detection with DNA probe hybridisation and amplification methods $[6,7]$. Significant differences in detection rates between cell culture and LCR for $C$. trachomatis detection have been recognised [8]. Testing of additional samples from the patient population may prove LCR to be more sensitive, but true benefits would have to be determined by completing patient outcome studies.

The ability to use LCR to test FVU for the presence of C. trachomatis is a true advantage of this assay. A number of clinical studies evaluating the efficacy of

Table 1. Results of FVU stability studies

\begin{tabular}{|c|c|c|c|c|c|c|c|c|}
\hline \multirow[b]{2}{*}{ Sample no. } & \multirow[b]{2}{*}{ Number of EBs* } & \multicolumn{7}{|c|}{$\mathrm{S} / \mathrm{CO}$ values } \\
\hline & & Initial & $1^{\dagger}$ & 2 & 3 & 4 & 5 & 6 \\
\hline 206 & 8 & 4.31 & 4.40 & 4.00 & 4.01 & 4.15 & 4.64 & 3.78 \\
\hline 270 & 8 & 3.89 & 3.78 & 3.91 & 4.55 & 2.93 & 3.06 & 3.89 \\
\hline 306 & 6 & 4.10 & 4.34 & 4.59 & 3.96 & 3.34 & 4.38 & 4.13 \\
\hline 334 & 1 & 3.14 & 2.38 & 3.53 & 2.32 & 2.76 & 3.94 & 3.11 \\
\hline 361 & 3 & 4.46 & 4.6 & 2.19 & 3.31 & 2.58 & $(0.53)$ & $(0.05)$ \\
\hline 438 & 3 & 4.21 & 3.61 & 1.66 & 2.63 & 1.15 & 2.62 & 2.06 \\
\hline 455 & 3 & 4.65 & 3.8 & 3.51 & 4.65 & 3.98 & 4.04 & 3.42 \\
\hline 491 & 0 & 1.74 & 2.32 & 2.12 & $(0.71)^{\ddagger}$ & 2.45 & 2.12 & 1.98 \\
\hline 511 & 4 & 2.95 & 2.93 & 3.36 & 2.35 & 1.93 & $(0.07)$ & $(0.05)$ \\
\hline
\end{tabular}

${ }^{*}$ Number of elementary bodies in $1.0 \mathrm{ml}$ urine as determined from cytospin preparation.

† Weekly intervals of testing after initial positive result.

$\ddagger($ ) indicates $\mathrm{S} / \mathrm{CO}$ value below positive cut-off point. 
testing FVU with LCR have shown sensitivities ranging from $86 \%$ to $96 \%$, with specificities approaching $100 \%$ when compared to an expanded 'gold-standard' of culture and DFA [9-14]. The high level of agreement between positive cervical and corresponding urine samples observed here is consistent with these reports [13]. The high rate of concordance in the results of paired cervical and urine samples reflects the value of testing FVU, which is a non-invasive approach to sample collection and, therefore, could result in greater numbers of patients being tested, an important component of chlamydial control programmes.

It appeared that low-level amplification occurred in the single negative urine sample collected from a patient with a positive cervical sample. It is possible that natural inhibitors, which have been described by others, were present in the sample and interfered with the assay $[8,11]$. Unfortunately, the sample was discarded before this could be confirmed by either diluting the sample and repeating the assay or by adding $C$. trachomatis DNA to the sample and attempting amplification. However, based on the fact that cervical samples from this patient tested positive by GP and LCR, we consider that this sample was indeed positive for $C$. trachomatis, and this represents a 'false-negative' result. This did not appear to be the case for the five samples with initial $\mathrm{S} / \mathrm{CO}$ values ranging from 0.34 to 0.74 , which were shown to be negative by repeat testing. The problem with these samples is likely to be in the detection portion of the assay. Non-specific binding of either unligated probe bearing the detection hapten or the antibody conjugate to substances (e.g., mucus) present in the sample could have resulted in increased fluorescence and elevated S/CO values. Cross-contamination between a positive and negative sample or from a contaminated work area is also a possibility, although less likely because of separation of work areas and stringent QA practices employed within this laboratory.

The data from the present study did not support the manufacturer's claim that properly collected and stored FVU samples are stable for testing only up to 4 days, unless frozen. While the sample volume was small and included only nine specimens, none of the positive samples reverted to negative until after storage for 3 weeks. This could be very beneficial to reference laboratories, which are more likely to adopt LCR testing and which are often faced with transportation delays due to travel distances, weekends, holidays, etc. More extensive study in the area of sample stability is needed for this assay. The sensitivity of testing midstream urine samples is another area for investigation, as current protocols for the collection of FVU are difficult for some health care providers to adapt to and may be confusing for some patients, as mid-stream urine is a more common collection request.

The high sensitivity of molecular amplification assays is not subject to dispute; however, the laboratory cost per patient result is typically high compared to other options available for direct specimen testing. This study did not demonstrate an increase in the number of positive samples detected by LCR compared to GP. While this observation may not continue over time with the testing of a larger number of samples, it must be taken into consideration when decisions are made relating to testing methodology. Other issues to consider include the patient population being served and the prevalence of infection within this group, as well as the clinical and social significance of missing an infection if a less sensitive assay is used.

We thank both Gen-Probe and Abbott Laboratories for providing the necessary collection kits and reagents needed to complete this study. We also thank the participating centres for enrolling patients and assisting in sample collection, and we would like to acknowledge Anne Bailey for her assistance in the preparation of this manuscript.

\section{References}

1. Centers for Disease Control. Recommendations for the prevention and management of Chlamydia trachomatis infections 1993. MMWR Morb Mortal Wkly Rep 1993; 42: (RR-12) $1-39$.

2. Schachter J. Chlamydial infections. West J Med 1990; 153: $523-534$.

3. Hillis S, Black C, Newhall J, Walsh C, Groseclose SL. New opportunities for Chlamydia prevention: applications of science to public health practice. Sex Transm Dis 1995; 22: 197-202.

4. Scholes D, Stergachis A, Heidrich FE, Andrilla H, Holmes KK, Stamm WE. Prevention of pelvic inflammatory disease by screening for cervical chlamydial infection. $N$ Engl $\mathrm{J} \mathrm{Med}$ 1996; 334: $1362-1366$.

5. Kluytmans JAJW, Neisters HGM, Mouton JW et al. Performance of nonisotopic DNA probe for detection of Chlamydia trachomatis in urogenital specimens. J Clin Microbiol 1991; 29: 2685-2689.

6. Peeling RW, Brunham RC. Molecular techniques for the laboratory identification of Chlamydia trachomatis. Am Environ Lab 1994; 6: 78-81.

7. Wu C-H, Lee M-F, Yin S-C, Yang D-M, Chang S-F. Comparison of polymerase chain reaction, monoclonal antibody based enzyme immunoassay, and cell culture for detection of Chlamydia trachomatis in genital specimens. Sex Transm Dis 1992; 19: 193-197.

8. Schachter J, Stamm WE, Quinn TC, Andrews WW, Burczak $\mathrm{JD}$, Lee $\mathrm{HH}$. Ligase chain reaction to detect Chlamydia trachomatis infection of the cervix. J Clin Microbiol 1994; 32: 2540-2543.

9. Ånestad G, Berdal BP, Scheel O et al. Screening urine samples by leukocyte esterase test and ligase chain reaction for chlamydial infections among asymptomatic men. $J$ Clin Microbiol 1995; 33: 2483-2484.

10. Bassiri M, Hu HY, Domeika MA et al. Detection of Chlamydia trachomatis in urine specimens from women by ligase chain reaction. $J$ Clin Microbiol 1995; 33: 898-900.

11. Chernesky MA, Lee $\mathrm{H}$, Schachter $J$ et al. Diagnosis of Chlamydia trachomatis urethral infection in symptomatic and asymptomatic men by testing first-void urine in a ligase chain reaction assay. $J$ Infect Dis 1994; 170: 1308-1311.

12. Chernesky MA, Jang D, Lee $\mathrm{H}$ et al. Diagnosis of Chlamydia trachomatis infections in men and women by testing first-void urine by ligase chain reaction. $J$ Clin Microbiol 1994; 32: 2682-2685.

13. Schachter J, Moncada J, Whidden $\mathrm{R}$ et al. Noninvasive tests for diagnosis of Chlamydia trachomatis infection: application of ligase chain reaction to first-catch urine specimens of women. J Infect Dis 1995; 172: 1411-1414.

14. van Doornum GJJ, Buimer $M$, Prins $M$ et al. Detection of Chlamydia trachomatis infection in urine samples from men and women by ligase chain reaction. J Clin Microbiol 1995; 33: $2042-2047$. 